\title{
Anti-Polar Ordering in Polycrystalline Samarium Doped $\mathrm{BiFeO}_{3}$
}

\author{
Andreja Bencan ${ }^{1}$, Goran Drazic ${ }^{2}$, Julian Walker ${ }^{1,3}$, Tadej Rojac ${ }^{1}$ and Barbara Malic ${ }^{1}$ \\ 1. Electronic Ceramics Department, Jozef Stefan Institute, Ljubljana, Slovenia. \\ 2. Laboratory for Materials Chemistry, National Institute of Chemistry, Ljubljana, Slovenia. \\ 3. The School of Materials Science and Engineering, University of New South Wales, Sydney, Australia.
}

Bismuth ferrite $\left(\mathrm{BiFeO}_{3}\right.$ or $\left.\mathrm{BFO}\right)$ has recently been intensively investigated due to its ability to exhibit a rare combination of both ferroelectric and antiferromagnetic ordering at room temperature [1]. Current literature shows that several solid solutions between $\mathrm{BFO}$ and rare-earth $(\mathrm{La}, \mathrm{Nd}, \mathrm{Sm}, \mathrm{Gd})$ ferrites, exhibit morphotropic phase boundary (MPB) between a polar (rhombohedral) and non-polar (orthorhombic) phase at which electromechanical properties are maximized [2]. The discovery of a compositionally induced anti-polar phase in the vicinity of the polar-to-non-polar phase transition stimulated further interest in the structural behavior of these BFO-based materials [3].

Whereas a number of structural studies on the atomic level coupled with the analysis of the boundaries between different phases and ferroelectric/ferroelastic domains have been done on thin films, those data on bulk ceramics are quite limited. In general, the preparation of $\mathrm{BFO}$ ceramic is not trivial. In addition to the problems related to the $\mathrm{Bi}_{2} \mathrm{O}_{3}$ volatility, the $\mathrm{BFO}$ easily reacts with different impurity elements, resulting in formation of undesired secondary phases [4]. Modification of BFO with RE oxides introduced an additional problem related with the distribution of the A-site $(\mathrm{Bi}, \mathrm{Sm})$ cations in the perovskite, which is rarely considered.

The aim of this study was to investigate the influence of the processing methods and conditions on the chemical homogeneity and the phase composition of $\mathrm{Bi}_{0.88} \mathrm{Sm}_{0.12} \mathrm{FeO}_{3}$ (Sm-BFO) ceramics on the atomic level, particularly on the formation of the reported anti-polar $\mathrm{PbZrO}_{3}$-like phase. The Sm-BFO ceramics were prepared using two different synthesis methods, i.e., a conventional solid-state synthesis (non-activated) and mechanochemically activated synthesis. Analytical transmission-electron microscopes - TEM (JEOL JEM 2010 and Cs-probe corrected JEM-ARM200CF) were employed for the atomistic structural studies.

In both $\mathrm{BFO}$ ceramics, the anti-polar phase with the perovskite unit cell quadrupled along the $\langle 110\rangle_{\mathrm{p}}$ and the $<001>_{\mathrm{p}}$ directions was detected (Fig. 1). In non-activated BFO ceramics, the anti-polar phase was found in up to 100-nm large grains in which the amount of Sm was higher compared to the BFO matrix. In activated BFO ceramics, the anti-polar phase was detected as small, nano-inclusions within the BFO matrix. In contrast to the non-activated BFO, in activated ceramics no detectable difference was observed in the chemical composition between inclusions and the matrix, which was probed with an atomic-level resolution. Off-centering of A- and B-site atoms was determined from the atomic resolution HAADF micrograph (Fig. 2). The origin of the anti-polar phase appearance and its atomically resolved structure in both BFO-modified ceramics will be discussed and related to the methods of synthesis.

References:

[1] G Catalan and JF Scott, Adv. Mater 21 [24] (2009), p.2463. 
[2] S Fujino et al, Appl. Phy. Lett. 92 [20] (2008), p.2002904.

[3] S Karimi et al, Appl. Phys. Lett. 94 (2009), p. 112903.

[4] M Valant et al, Chem. Mater. 19 (2007), p.5431.

[5] The authors would like to thank Brigita Kmet for help in TEM sample preparation. Maja Makarovic is acknowledged for the preparation of the ceramics. The work was carried out within the research program "Electronic Ceramics, Nano, 2D and 3D Structures" P2-0105 and within the project "High Performance Piezoelectric Materials for Sensors and Actuators in High-Temperature Applications" J2-5483 (Slovenian Research Agency).
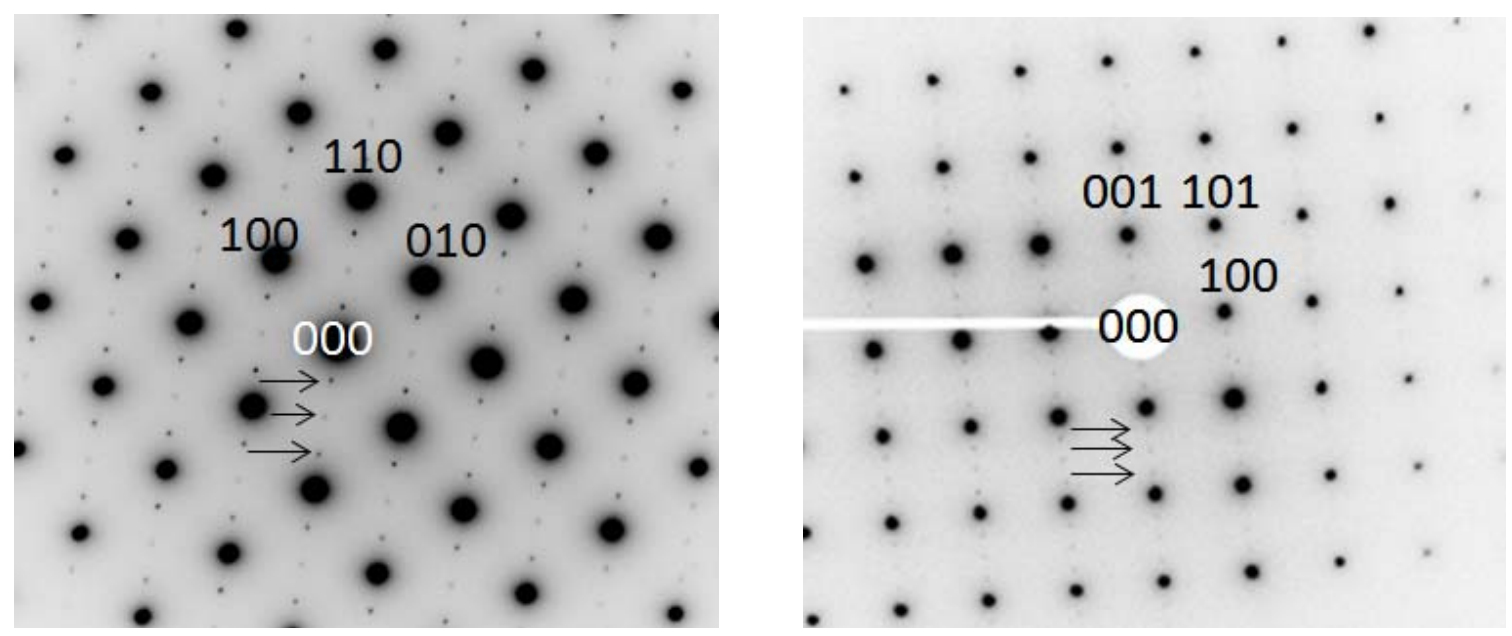

Figure 1. Two SAED patterns from anti-polar phase, quadrupled in [hk0] direction observed in $[001]_{\mathrm{p}}$ zone axis and quadrupled in [001] direction in [010] zone axis. $1 / 4(\mathrm{hk} 0)$ and $1 / 4(001)$ reflections are indicated by arrows.

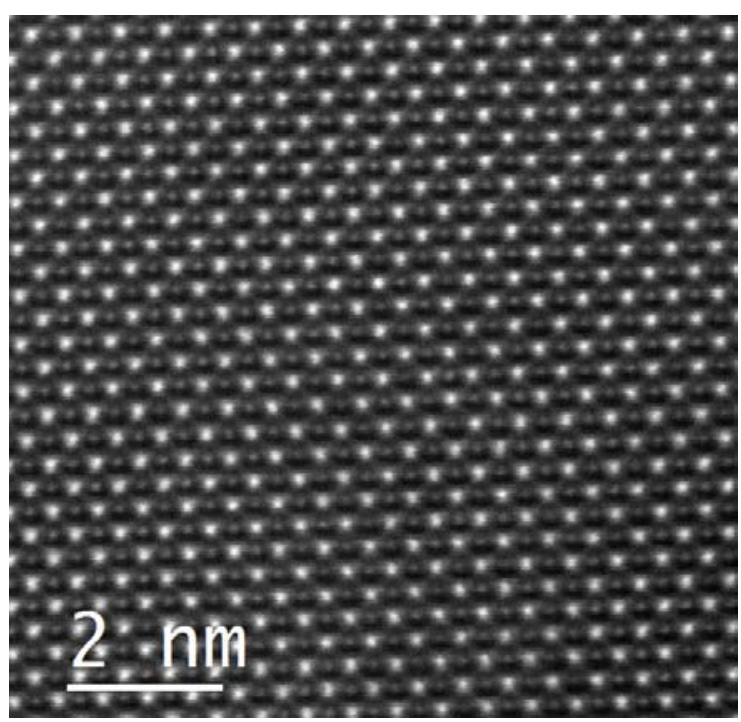

Figure 2. Atomic resolution HAADF STEM image of anti-polar phase in Sm-BFO, prepared by mechanochemically activated synthesis in the [001]p zone axis where $1 / 4(\mathrm{hk} 0)$ reflections were present. 